\title{
Low-emittance beam injection for a synchrotron radiation source using an X-ray free-electron laser linear accelerator
}

\author{
Toru Hara $\odot,{ }^{1, *}$ Tsuyoshi Aoki, ${ }^{2}$ Hideki Dewa, ${ }^{2}$ Takahiro Fujita, ${ }^{2}$ Kenji Fukami, ${ }^{2}$ Toru Fukui, ${ }^{1}$ \\ Toshihiko Hiraiwa, ${ }^{1}$ Naoyasu Hosoda,${ }^{2}$ Takahiro Inagaki, ${ }^{1}$ Toshiro Itoga,${ }^{2}$ Eito Iwai, ${ }^{2}$ \\ Akio Kiyomichi, ${ }^{2}$ Chikara Kondo, ${ }^{2}$ Hirokazu Maesaka, ${ }^{1}$ Mitsuhiro Masaki, ${ }^{2}$ \\ Shinichi Matsubara, ${ }^{2}$ Osamu Morimoto, ${ }^{3}$ Takashi Ohshima, ${ }^{2}$ Masaya Oishi, ${ }^{2}$ Masazumi Shoji, ${ }^{2}$ \\ Kouichi Soutome, ${ }^{2}$ Yasuyuki Tajiri, ${ }^{3}$ Shiro Takano, ${ }^{2}$ Kazuhiro Tamura, ${ }^{2}$ \\ Yukiko Taniuchi, ${ }^{2}$ Kazuaki Togawa, ${ }^{1}$ Takahiro Watanabe, ${ }^{2}$ \\ Toshinori Yabuuchi, ${ }^{2}$ and Hitoshi Tanaka ${ }^{1}$ \\ ${ }^{1}$ RIKEN SPring-8 Center, Kouto 1-1-1, Sayo, Hyogo 679-5148, Japan \\ ${ }^{2}$ Japan Synchrotron Radiation Research Institute, Kouto 1-1-1, Sayo, Hyogo 679-5198, Japan \\ ${ }^{3}$ SPring-8 Service Co., Ltd., Kouto 1-20-5, Shingu-cho, Tatsuno, Hyogo 679-5165, Japan
}

\section{(Received 13 August 2021; accepted 11 October 2021; published 22 November 2021)}

\begin{abstract}
The full-energy beam injection system from SACLA to the SPring-8 storage ring has been developed. Low-emittance beam injection is required for the future upgrade of SPring- 8 due to its small injection beam aperture. The electron beam injection from an X-ray free-electron laser linear accelerator not only satisfies the emittance requirement but also it confers a cost benefit compared to a conventional dedicated injector system. In SPring-8, the SACLA linear accelerator is now delivering X-ray free-electron laser photon beams and serving as a full-time injector of SPring- 8 in parallel.
\end{abstract}

DOI: $10.1103 /$ PhysRevAccelBeams.24.110702

\section{INTRODUCTION}

A small dynamic aperture for injected electron beams becomes an issue in recent upgrade of storage ring-based light sources pursuing low-emittance toward a diffraction limit [1-3]. It is due to nonlinearity caused by strong focusing magnets necessary in a multibend optics design. Some ideas have been proposed to improve injection efficiency, such as longitudinal or swap-out on axis injection [4-7]. We choose, however, a conventional off axis injection scheme using kicker and in-vacuum septum magnets in SPring-8-II, which is the upgrade project of SPring-8 $[8,9]$. Instead, the emittance of the injected beam is significantly reduced by using a low-emittance linear accelerator of an X-ray free-electron laser (XFEL).

In 2011, the XFEL facility SPring-8 Angstrom Compact free-electron LAser (SACLA) was constructed in the SPring-8 campus [10]. SACLA is located next to the SPring-8 storage ring and an XFEL to Storage ring Beam Transport line (XSBT) connects the end of the

\footnotetext{
*toru@spring8.or.jp
}

Published by the American Physical Society under the terms of the Creative Commons Attribution 4.0 International license. Further distribution of this work must maintain attribution to the author(s) and the published article's title, journal citation, and DOI.
SACLA linear accelerator to the exit of the existing $8 \mathrm{GeV}$ booster synchrotron (Fig. 1).

Ahead of the upgrade project, the full-energy beam injection from SACLA had been tested since 2019 using the present SPring- 8 storage ring. In addition to the low emittance, utilization of SACLA as an injector brings an economical advantage. In case of a conventional dedicated injector system, accelerators are always kept in operation for top-up injection, and the electron beam is injected only a few times per minute with consuming electricity at most of the time just for idling the accelerators. On the other hand, the accelerator of SACLA is already in operation to deliver XFEL photon beams to users, and no extra electricity is required for the beam injection. In the case of SPring-8, the electricity consumption can be reduced by $30 \%$ and the maintenance cost can be cut down by shutting

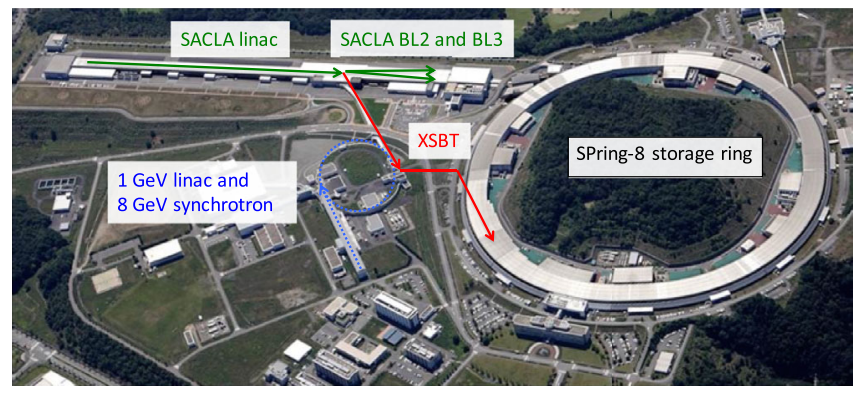

FIG. 1. Bird's-eye view of the SPring-8 campus. 


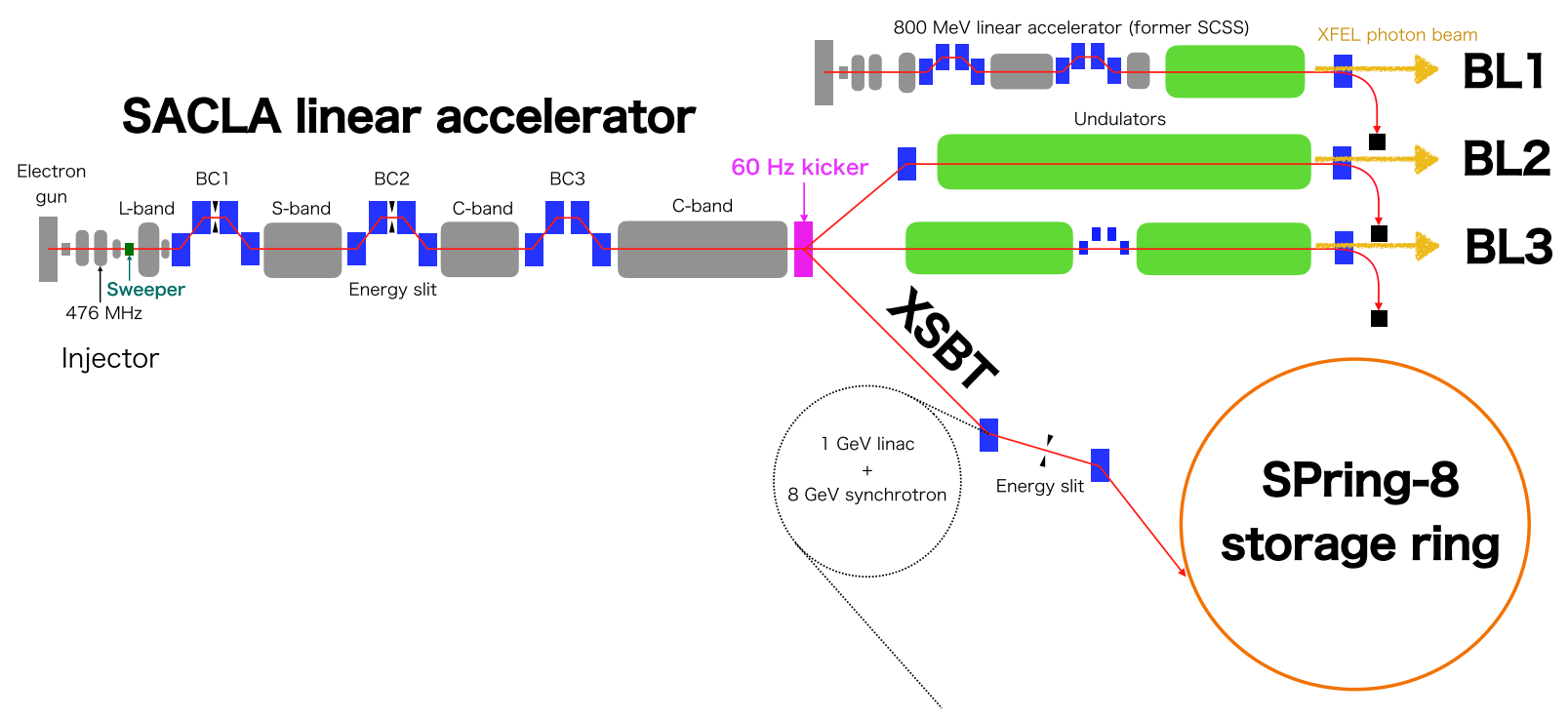

FIG. 2. Schematic layout of SACLA and XSBT.

down the more than twenty-year-old injector system consisting of a $1 \mathrm{GeV}$ linear accelerator and an $8 \mathrm{GeV}$ synchrotron.

\section{SACLA BEAM INJECTION SCHEME}

Figure 2 is a schematic of the SACLA facility. Currently, three FEL beam lines are in operation. BL1 is a soft $\mathrm{x}$-ray FEL beam line driven by a dedicated $800 \mathrm{MeV}$ linear accelerator (the former SPring-8 Compact SASE Source (SCSS) test accelerator), and BL2 and BL3 are XFEL beam lines [11,12]. The destination of the electron beam is switched from pulse to pulse using a kicker magnet installed at the end of the SACLA linear accelerator [13]. The beam repetition rate is $60 \mathrm{~Hz}$ and the electron beam is delivered to either the two XFEL beam lines or XSBT for the beam injection.

While the beam is injected to the storage ring at a fixed energy of $8 \mathrm{GeV}$, the beam energy for the XFEL operation changes depending on user experiments. The parameters of the electron beam are summarized in Table I. In order to independently control the electron beam parameters of each destination, a multienergy operation scheme was developed, in which the number of $\mathrm{rf}$ accelerator cavities and rf phases are changed from pulse to pulse [14].

To achieve the pulse-by-pulse control of the destination and the parameters of accelerator components, pattern tables for a period of $1 \mathrm{~s}$ are prepared and stored in a master controller $[15,16]$. Each table contains destinations for 60 electron bunches corresponding to a beam repetition of $60 \mathrm{~Hz}$, and the master controller sends the destination of the next pulse to all accelerator components through a reflective memory network. Once the component receives the destination, it runs with prestored parameters of each beam destination, such as an rf phase and a deflection angle of the kicker magnet. When the beam injection is requested from the storage ring for top-up injection, the table including the destination of XSBT is loaded to the master controller and the beam is injected to the storage ring in the next second.

Since the reference clock frequencies of the SACLA linear accelerator $(238 \mathrm{MHz})$ and the SPring- 8 storage ring $(508.58 \mathrm{MHz})$ are not related by an integer multiple, the beam timing is adjusted each time of the beam injection. The beam timing of SACLA is generated from a $238 \mathrm{MHz}$ master oscillator and a $60 \mathrm{~Hz}$ power line, so the maximum

TABLE I. Electron beam parameters for the XFEL operation and the beam injection.

\begin{tabular}{lcc}
\hline \hline & XFEL operation & Beam injection (at the injection point) \\
\hline Destination & BL2 or BL3 & XSBT \\
Beam energy & $4-8 \mathrm{GeV}$ & $8 \mathrm{GeV}$ \\
Bunch length (FWHM) & $<20 \mathrm{fs}$ & $>5 \mathrm{ps}$ (estimated) \\
Bunch charge & $200 \mathrm{pC}$ & $200 \mathrm{pC}$ \\
Emittance (projected) & $\sim 150 \mathrm{pm} \mathrm{rad}$ & $\sim 1 \mathrm{~nm} \mathrm{rad}$ (estimated) \\
Energy spread (rms, projected) & $\leq 1 \times 10^{-3}$ & $\sim 1 \times 10^{-3}$ (estimated) \\
Repetition rate & $60 \mathrm{~Hz}$ maximum & $10 \mathrm{~Hz}$ for accumulation \\
& & $<1 \mathrm{~Hz}$ for top up \\
\hline \hline
\end{tabular}




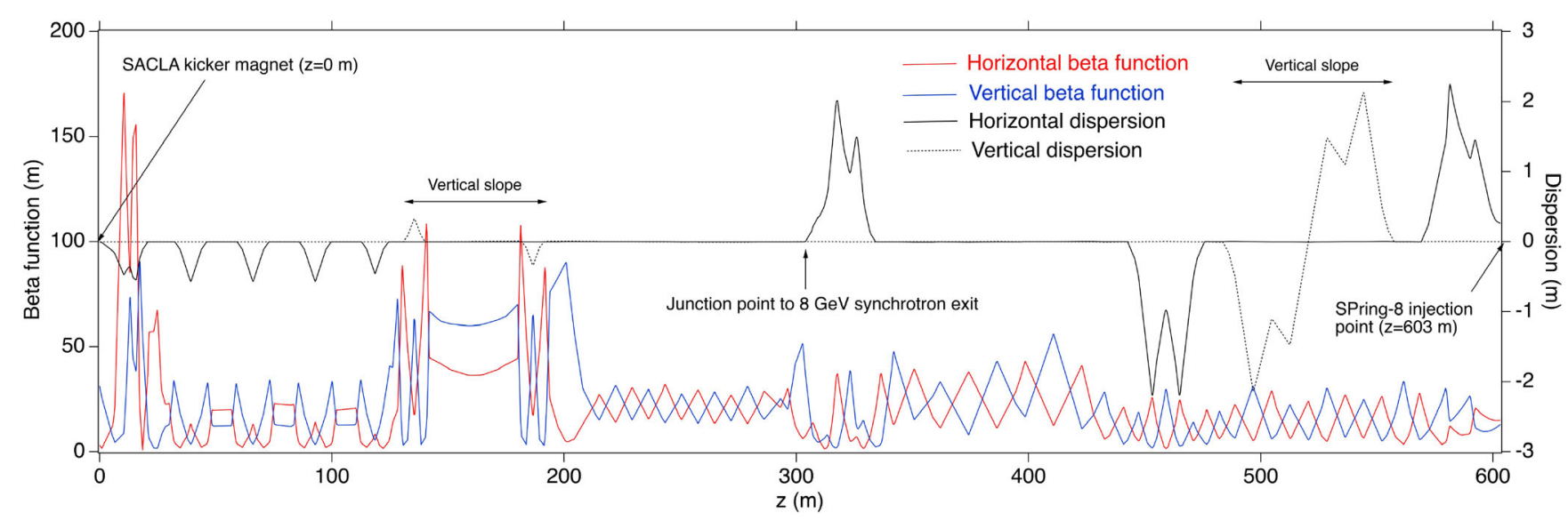

FIG. 3. Beta functions and dispersion functions of XSBT from the SACLA kicker magnet to the beam injection point of the SPring-8 storage ring. Horizontal and vertical beta functions are shown in red and blue solid lines, and horizontal and vertical dispersion functions are shown in black solid and dotted lines.

time difference with respect to a $208.8 \mathrm{kHz}$ revolution frequency of SPring- 8 is $2.1 \mathrm{~ns}$. The synchronization between the beam timing of SACLA and the target rf bucket of SPring-8 is achieved by two steps. First, a trigger signal, which is generated $15.5 \mathrm{~ms}$ before the beam timing, is delayed up to 40 turns of the storage ring $(<197 \mu$ s) to reduce the time difference within 105 ps. As a second step, the frequency of $238 \mathrm{MHz}$ is modulated to further reduce the time difference and finely synchronize the two accelerators. The developed timing system attains synchronization with an accuracy of $3.8 \mathrm{ps}$ (rms), which is small enough compared to the SPring-8 electron bunch length of 10 ps (rms) [17].

\section{BEAM INJECTION TO SPRING-8}

Figure 3 shows the electron beam optics of XSBT. The first half of XSBT, which is newly built to connect SACLA to the exit of the synchrotron, is based on a double bend achromatic (DBA) lattice. The existing transport line from the synchrotron to the storage ring is reused as the last half of XSBT, whose lattice is based on FODO.

For the accumulation from $0 \mathrm{~mA}$, SACLA injects the beam at $10 \mathrm{~Hz}$ and it takes about 10 min to reach $100 \mathrm{~mA}$, which is the nominal current of SPring-8. During the $10 \mathrm{~Hz}$ injection, XFEL operation is suspended. Once the storage ring is filled up, the stored current is maintained by the topup injection performed in parallel with the XFEL operation. Figure 4 shows the stored current of the SPring-8 storage ring during the $10 \mathrm{~Hz}$ injection followed by the top-up injection. The electron bunch charge of SACLA is around 200 pC. In Fig. 4, the electron beam is injected twice a minute under the condition of insertion device gaps being closed. When the beam is injected to the storage ring, two pulses, which are the injection pulse and a blank pulse for hysteresis correction as described later, are missing in the XFEL operation.
While the injection efficiency of $100 \%$ is achieved with all insertion device gaps open, the efficiency is slightly reduced to around $90 \%$ under the condition of daily user operation gaps. The reduction of the injection efficiency may come from a large energy chirp of the SACLA electron beam.

Figure 5 compares the beam profiles of SACLA and the $8 \mathrm{GeV}$ synchrotron using a screen monitor located close to the injection point of the SPring-8 storage ring [18]. Since the emittance of the synchrotron is $200 \mathrm{~nm}$ rad and twice larger than the transverse acceptance of the storage ring [Fig. 5(b)], the electron beam was usually collimated for better injection efficiency [Fig. 5(c)] $[19,20]$. The expected emittance of the electron beam from SACLA is around $1 \mathrm{~nm} \operatorname{rad}$ [Fig. 5(a)], which is significantly smaller than that of the synchrotron by more than two orders of magnitude.

The influence of the beam injection on the XFEL operation is carefully investigated. To switch the XFEL beam lines, the kicker magnet current alternates zero (BL3) and a fixed negative value (BL2) with a magnetic field stability of $10^{-5}$ [21]. But once the kicker magnet is flipped

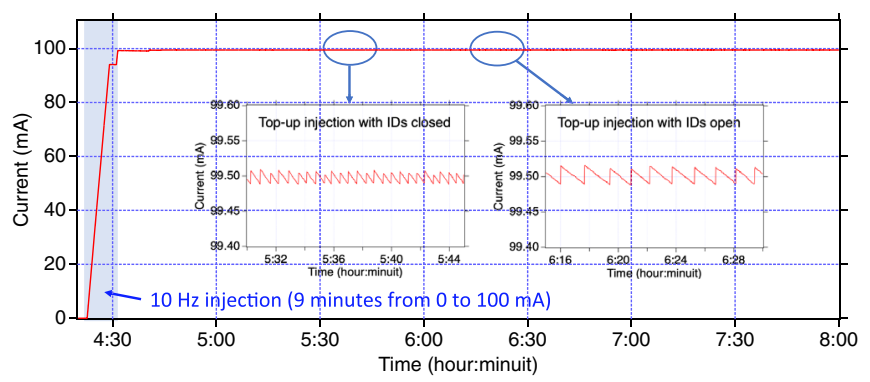

FIG. 4. Stored current of the SPring-8 storage ring during the beam injection. The electron beam is injected from SACLA at $10 \mathrm{~Hz}$ from $0 \mathrm{~mA}$ and the current is maintained at $100 \mathrm{~mA}$ with the top-up injection. Insets show the stored current during the top-up injection. 

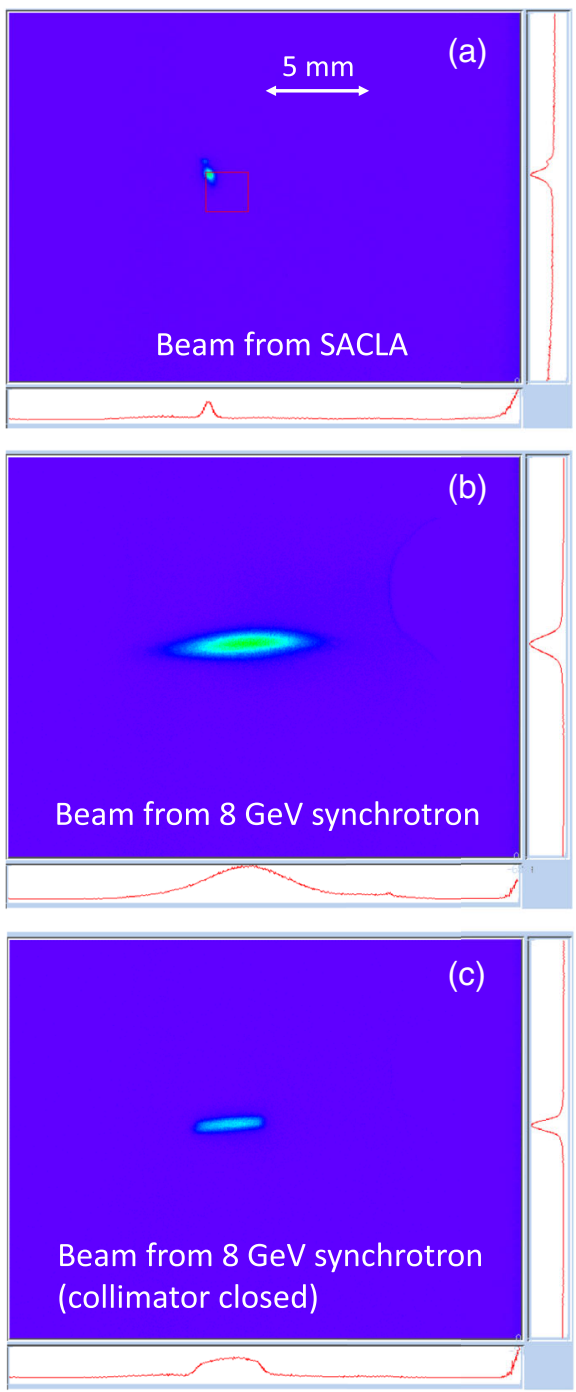

FIG. 5. Transverse beam profiles observed on a screen monitor close to the beam injection point of the SPring- 8 storage ring. Injected beams from SACLA (a) and the $8 \mathrm{GeV}$ synchrotron (b, c) are shown. In (c), a collimator installed at the synchrotron exit is closed to improve injection efficiency.

to a positive current (XSBT) for the beam injection, a hysteresis effect of the magnet yoke is observed, which results in orbit deviation in the XFEL undulators and deteriorates the pointing stability of the XFEL photon beams, particularly at BL3. The material of the yoke is laminated silicon steel plates with $0.35 \mathrm{~mm}$ thickness.

In order to remove the hysteresis effect, the kicker magnet is excited with a negative blank pulse without the beam right after the positive pulse of the beam injection. Figure 6 shows the pointing stability of the XFEL photon beam measured at the BL3 beam line station located $40 \mathrm{~m}$ downstream of the undulator end. The center positions of the photon beam are measured for $6 \mathrm{~h}$ during the top-up injection and histograms are plotted. In Fig. 6, black lines indicate the photon beam position without the beam
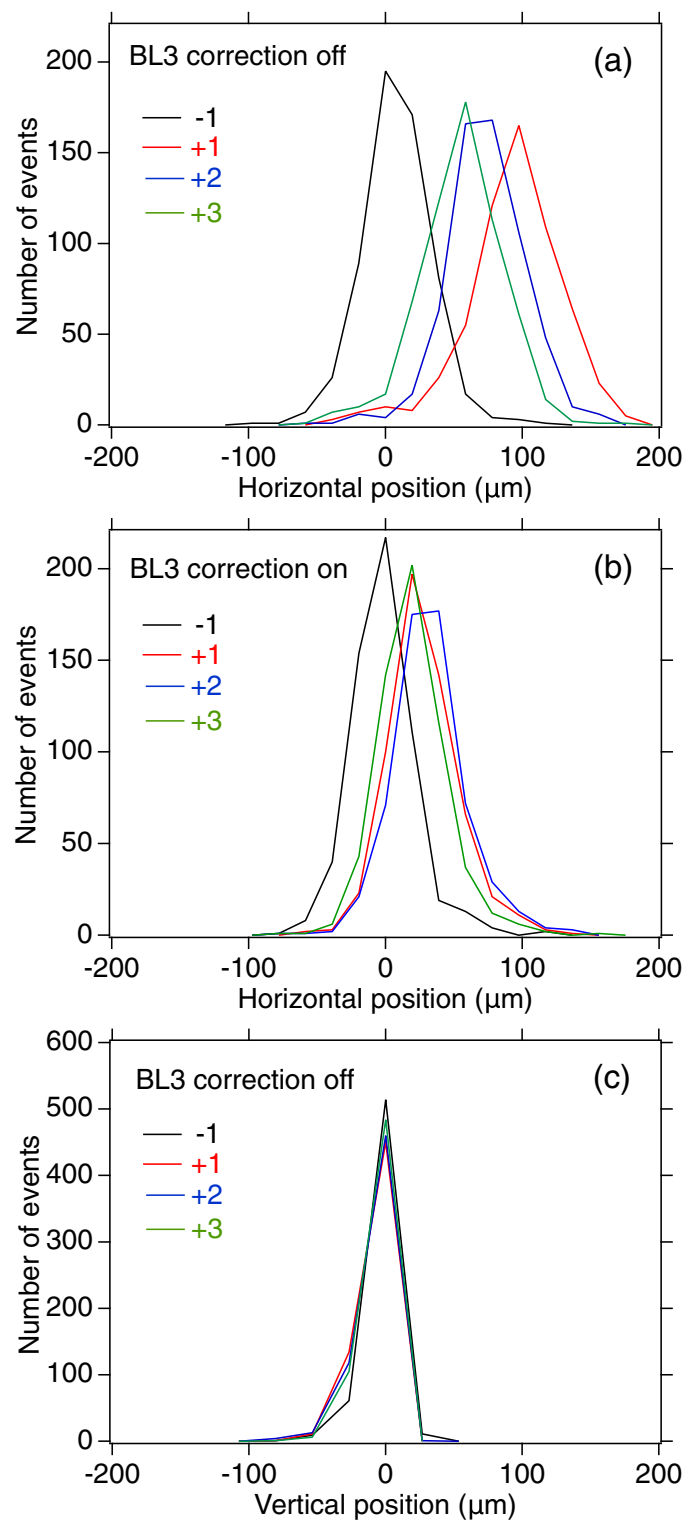

FIG. 6. Pointing stability of the XFEL photon beam observed at $40 \mathrm{~m}$ downstream of the BL3 undulator end. The data are collected for $6 \mathrm{~h}$ under the top-up injection. Black lines correspond to the last pulse before the injection, and red, blue, and green lines correspond to the first, second, and third pulses after the injection. Histograms of the horizontal beam position are plotted for without (a) and with (b) hysteresis correction. The kicker yoke hysteresis does not affect vertical stability as shown in (c).

injection, and red, blue, and green lines are the positions of three successive pulses immediately after the beam injection respectively. As shown in Fig. 6(a), even applying the blank pulse, small magnetization still remains. The horizontal position jumps by $100 \mu \mathrm{m}$ after the injection and then gradually comes back. In order to reduce this movement, the kicker magnet currents of these three pulses are corrected in Fig. 6(b). Note that the photon beam sizes are 


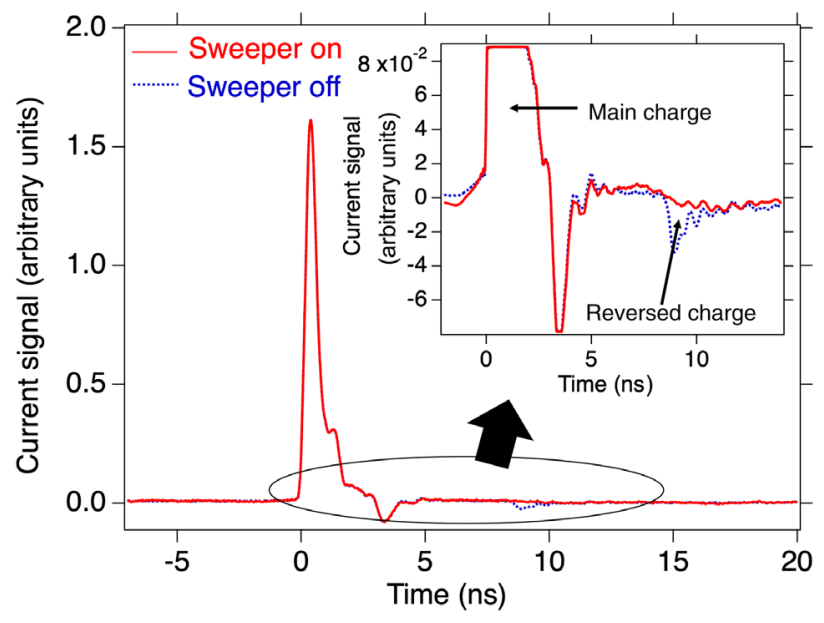

FIG. 7. Current monitor signals measured between the $476 \mathrm{MHz}$ rf cavity and the L-band accelerator of the injector section. Red solid and blue dotted lines correspond to sweeper on and off, respectively. An enlarged view is inserted to show the reversed charge having opposite polarity at $9 \mathrm{~ns}$ after the main charge.

about $500 \mu \mathrm{m}$ (FWHM) in both horizontal and vertical directions and there is no hysteresis effect vertically.

For some time-resolved experiments of synchrotron radiation facilities, such as nuclear resonance scattering, a high-contrast ratio of bunch charges is necessary between electron injected and adjacent noninjected empty $\mathrm{rf}$ buckets. At SPring-8, a ratio of $10^{-8}-10^{-10}$ is typically requested for the empty buckets [22]. In the beam injection from SACLA, however, it turned out that a small number of electrons are detected at the ninth bucket after the injected rf bucket.

SACLA employs a thermionic cathode gun with an initial bunch length of $1 \mathrm{~ns}$ after a beam chopper. Although the electron bunch is longitudinally compressed by velocity bunching in the injector section, it has a long tail after a charge concentrated core [23]. As a result, some electrons at the tail are captured in deceleration rf phases and reversed in an L-band accelerator, which is installed at the end of the injector section. Figure 7 is beam current signals measured between the L-band accelerator and a $476 \mathrm{MHz}$ rf cavity located $1.8 \mathrm{~m}$ upstream of the L-band accelerator. After the main charge, which is about $1 \mathrm{nC}$ and moving downstream, a reversed charge of about $10 \mathrm{pC}$ is observed with opposite polarity meaning that it moves upstream (a blue dotted line in Fig. 7). The reversed charge is then accelerated and turned around again in the $476 \mathrm{MHz}$ cavity. The round-trip time of the reversed electrons between the L-band accelerator and the $476 \mathrm{MHz}$ cavity is about $18 \mathrm{~ns}$ corresponding to nine times the $508 \mathrm{MHz}$ rf bucket interval of the storage ring. Once the reversed electrons are injected to the ninth bucket after the target $\mathrm{rf}$ bucket, they have a significantly long lifetime due to lowcharge density and do not decay. Therefore, the contrast ratio gradually worsens during the top-up injection.
In order to avoid the reversed electrons being injected into unwanted rf buckets of the storage ring, an electron sweeper is introduced between the $476 \mathrm{MHz}$ cavity and the L-band accelerator (Fig. 2). It kicks out the reversed electrons using pulsed electric fields. Together with the use of energy slits at dispersive locations, the charge contrast ratio is improved roughly by two orders of magnitude, and $10^{-8}-10^{-9}$ is routinely obtained even after several days of the top-up injection. In addition, an electron bunch knockout system is developed and installed in the storage ring, which applies rf fields to unwanted electron bunches and excites vertical betatron oscillations. The vertically deflected electrons are removed at a vertical scraper. When users request a high contrast ratio, the bunch knockout is performed several times a day to maintain the contrast ratio at the order of $10^{-10}$.

\section{EMITTANCE GROWTH IN BEAM TRANSPORT LINE}

Although the electron beam injection from SACLA has achieved much smaller emittance compared to that from the synchrotron as shown in Fig. 5, the emittance growth in XSBT cannot be ignored mainly due to quantum excitation of synchrotron radiation (SR) and second-order dispersion.

The emittance growth $\left(\Delta \epsilon_{x, y}\right)$ attributed to quantum excitation can be written in the horizontal $(x)$ or vertical $(y)$ plane as

$$
\Delta \epsilon_{x, y}=\frac{55 r_{e} \hbar \gamma^{5}}{48 \sqrt{3} m_{e} c} \int \frac{\mathcal{H}_{x, y}(z)}{\rho_{x, y}^{3}(z)} d z
$$

where the integration is taken along the beam trajectory $z . r_{e}$, $\hbar, \gamma, m_{e}$, and $c$ are the classical electron radius, the Dirac's constant, the Lorentz factor of the electron beam, the electron rest mass, and the speed of light in vacuum, respectively. $\mathcal{H}_{x, y}$ is defined as $\mathcal{H}_{x, y}=\beta_{x, y} \eta_{x, y}^{\prime 2}+2 \alpha_{x, y} \eta_{x, y} \eta_{x, y}^{\prime}+\gamma_{x, y} \eta_{x, y}^{2}$ with Twiss parameters $\left(\alpha_{x, y}, \beta_{x, y}, \gamma_{x, y}\right)$ and linear dispersion functions $\left(\eta_{x, y}, \eta_{x, y}^{\prime}\right) . \rho_{x, y}$ is the bending radius [24,25].

There are 26 horizontally deflecting and 6 vertically deflecting dipole magnets in the $600 \mathrm{~m}$-long XSBT, whose bending radii are around $20 \mathrm{~m}$ with deflection angles of $5-9^{\circ}$. The total energy loss due to SR in these dipole magnets is about $0.1 \%$ of $8 \mathrm{GeV}$, and the emittance growth obtained from Eq. (1) is in the order of hundreds pm rad. While the quantum excitation is negligible for the electron beam with large emittance like that of the synchrotron, it gives a severe impact on the small emittance beam of SACLA, whose typical projected emittance at the exit of the accelerator is $150 \mathrm{pm} \mathrm{rad}$.

In storage rings, the emittance is determined from the equilibrium condition of the quantum excitation and radiation damping. The emittance decrease due to the radiation damping is described as 

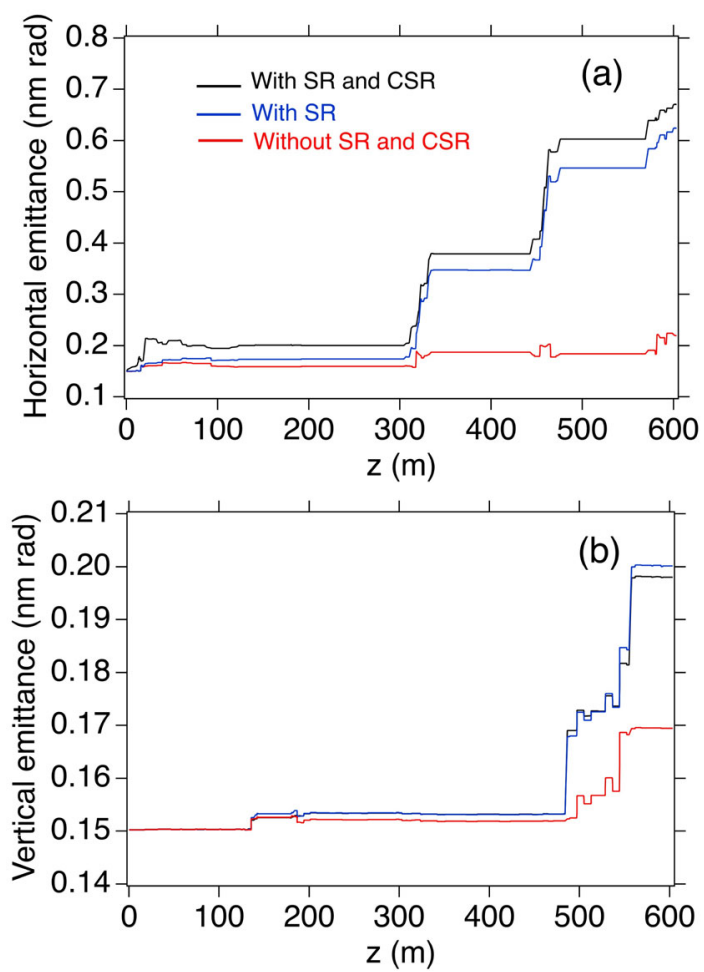

FIG. 8. Projected emittance growth along XSBT in the horizontal (a) and vertical (b) planes. Emittance is not normalized and the contribution of linear dispersion is subtracted. The calculation results are shown for three conditions: including SR and CSR effects (black lines), only SR effect (blue lines) and none of them (red lines). The initial beam has an $8 \mathrm{GeV}$ beam energy, $150 \mathrm{pm}$ rad emittance, a 20 fs bunch length (FWHM), $0.1 \%$ (rms) energy spread and a $200 \mathrm{pC}$ bunch charge.

$$
\Delta \epsilon_{x, y} \cong-\frac{2 r_{e} \gamma^{3}}{3} \int \frac{\epsilon_{x, y}(z)}{\rho_{x, y}^{2}(z)} d z
$$

where $\epsilon_{x, y}$ is the emittance in the horizontal or vertical plane [24]. Comparing Eqs. (1) and (2) for the case of the small emittance beam of $150 \mathrm{pm}$ rad passing through XSBT, it is found that the radiation damping is three orders of magnitude smaller than the quantum excitation and almost negligible.

Figure 8 is the calculated emittance growth along XSBT with the initial conditions assuming the SACLA electron beam of an $8 \mathrm{GeV}$ beam energy, $150 \mathrm{pm}$ rad emittance, a 20 fs bunch length (FWHM), 0.1\% (rms) energy spread, and a $200 \mathrm{pC}$ bunch charge. The calculations are performed using the ELEGANT code and the contribution of linear dispersion is subtracted in Fig. 8 [26].

As mentioned, the last half of XSBT $(z=300-600 \mathrm{~m})$ is the reuse of a nearly twenty-five-year-old transport line based on an FODO lattice with large dispersion functions. Therefore, $\mathcal{H}_{x, y}(z)$ in Eq. (1) becomes large resulting in severe emittance growth in the last half of XSBT (blue lines in Fig. 8). On the other hand, the effect of coherent

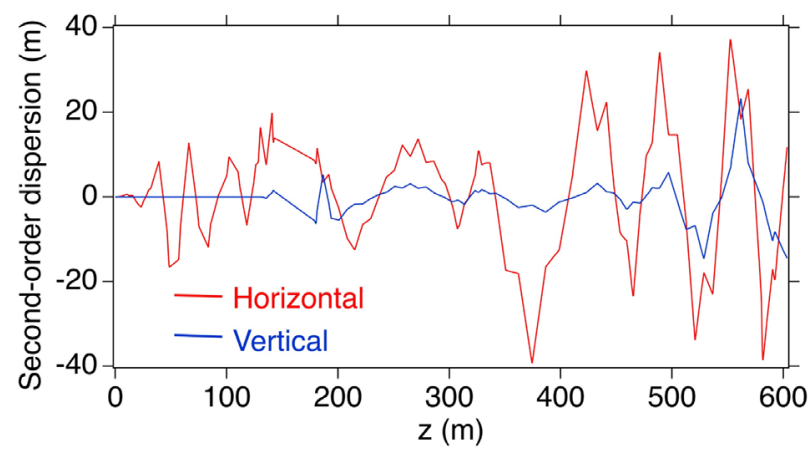

FIG. 9. Second-order geometrical dispersion functions of XSBT in the horizontal (red line) and vertical (blue line) planes.

synchrotron radiation (CSR) is rather limited (black lines in Fig. 8), because the electron bunch immediately lengthens to longer than 100 fs (FWHM) due to large $R_{56}$ (longitudinal linear dispersion) at the first part of XSBT and it reaches 8 ps at the end.

In Fig. 8, a gaussian energy spread is assumed, but the electron beam of SACLA has usually an energy-increasing chirp from head to tail of the bunch. In this case, the electron beam lengthens more quickly due to positive $R_{56}$ of XSBT, which is opposite to chicane bunch compressors, and the emittance growth becomes less important. Since the electron beam having an energy-decreasing chirp is first compressed and then stretched in XSBT, the emittance growth worsens due to the CSR effect.

The red lines of Fig. 8 are the emittance calculated without the electron energy change caused by SR or CSR. Nevertheless, the emittance increases by $50 \%$ in the horizontal plane and $15 \%$ in the vertical plane. There is no sextupole magnets installed in XSBT and this emittance increase is caused by second-order dispersion. Figure 9 shows the second-order geometrical dispersion functions of XSBT.

\section{SUMMARY}

The XFEL linear accelerator has been successfully used as a low-emittance injector of the SPring-8 storage ring since September 2020. The obtained emittance of $\sim 1 \mathrm{~nm}$ rad at the injection point is small enough for the future SPring-8-II, whose requirement is of the order of $\sim 10 \mathrm{~nm}$ rad. SACLA is now used as a full-time injector of SPring- 8 and the existing injector system was shut down in March 2021.

In the transport of a small emittance beam, the emittance growth due to quantum excitation should be taken into account in addition to CSR effects. To maintain small emittance in a transport line, it is important to control linear and nonlinear dispersion to reduce quantum excitation and avoid the emittance growth.

Although the present emittance growth in XSBT is acceptable for SPring-8-II, further emittance reduction would be possible by correcting second-order dispersion using sextupole magnets and optimizing initial energy 
spread. However, magnet rearrangement of the last half of $\mathrm{XSBT}$ is necessary to drastically reduce the emittance.

\section{ACKNOWLEDGMENTS}

The authors wish to acknowledge the support of the SACLA team. We are deeply grateful to the SPring-8 and SACLA operators for their help with the software development and accelerator operation.

[1] P. F. Tavares et al., Commissioning and first-year operational results of the MAX IV $3 \mathrm{GeV}$ ring, J. Synchrotron Radiat. 25, 1291 (2018).

[2] P. Raimondi, The ESRF low emittance upgrade, in Proceedings of IPAC2016 (Busan, Korea, 2016), p. 2023.

[3] L. Liu et al., Preliminary SIRIUS commissioning results, in Proceedings of IPAC2020 (Caen, France, 2020), p. 11.

[4] M. Aiba, M. Böge, F. Marcellini, Á. S. Hernández, and A. Streun, Longitudinal injection scheme using short pulse kicker for small aperture electron storage ring, Phys. Rev. ST Accel. Beams 18, 020701 (2015).

[5] S. Jiang and G. Xu, On-axis injection scheme based on a triple-frequency rf system for diffraction-limited storage ring, Phys. Rev. Accel. Beams 21, 110701 (2018).

[6] K. Harkay et al., High-charge injector for on-axis injection into a high-performance storage ring light source, in Proceedings of IPAC2019 (Melbourne, Australia, 2019), p. 3423.

[7] A. Xiao and M. Borland, Transport line design and injection configuration optimization for the Advanced Photon Source upgrade, in Proceedings of IPAC2018 (Vancouver, Canada, 2018), p. 1287.

[8] H. Tanaka et al., SPring-8 upgrade project, in Proceedings of IPAC2016 (Busan, Korea, 2016), p. 2867.

[9] S. Takano et al., Renovation of off-axis beam injection scheme for next-generation photon sources, in Proceedings of IPAC2019 (Melbourne, Australia, 2019), p. 2318.

[10] T. Ishikawa et al., A compact X-ray free-electron laser emitting in the sub-ångström region, Nat. Photonics 6, 540 (2012).

[11] T. Shintake et al., A compact free-electron laser for generating coherent radiation in the extreme ultraviolet region, Nat. Photonics 2, 555 (2008).

[12] S. Owada et al., A soft X-ray free-electron laser beamline at SACLA: The light source, photon beamline and experimental station, J. Synchrotron Radiat. 25, 282 (2018).
[13] T. Hara et al., Pulse-bypulse multi-beamline operation for x-ray free-electron laser, Phys. Rev. Accel. Beams 19, 020703 (2016).

[14] T. Hara et al., Time-interleaved multienergy acceleration for an X-ray free-electron laser facility, Phys. Rev. Accel. Beams 16, 080701 (2013).

[15] T. Fukui et al., The design of the control system for the SACLA/SPring-8 accelerator complex to use the linac of SACLA for a full-energy injector of SPring-8, in Proceedings of IPAC2019 (Melbourne, Australia, 2019), p. 2529.

[16] H. Maesaka et al., On-demand beam route and RF parameter switching system for time-sharing of a linac for x-ray free-electron laser as an injector to a 4thgeneration synchrotron radiation source, in Proceedings of IPAC2019 (Melbourne, Australia, 2019), p. 3427.

[17] T. Ohshima, H. Maesaka, N. Hosoda, and S. Matsubara, Timing synchronization system for beam injection from the SACLA linac to the SPring-8 storage ring, in Proceedings of IPAC2019 (Melbourne, Australia, 2019) p. 3882.

[18] S. Takano, M. Masaki, T. Masuda, and A. Yamashita, OTR based monitor of injection beam for top-up operation of the SPring-8, in Proceedings of DIPAC2005 (Lyon, France, 2005), p. 72.

[19] K. Fukami et al., Ejected beam emittance of the SPring-8 booster synchrotron, in Proceedings of EPAC2002 (Paris, France, 2002), p. 1888.

[20] K. Fukami et al., Beam collimation system for the SPring-8 top-up operation, in Proceedings of APAC2004 (Gyeongju, Korea, 2004), p. 103.

[21] C. Kondo et al., A stable pulsed power supply for multibeamline XFEL operations, Rev. Sci. Instrum. 89, 064704 (2018).

[22] K. Tamura and T. Aoki, Single bunch purity during SPring-8 storage ring top-up operation, in Proceedings of the 1st Annual Meeting of Particle Accelerator Society of Japan and the 29th Linear Accelerator Meeting in Japan (in English) (Funabashi, Japan, 2004), p. 581.

[23] T. Asaka et al., Low-emittance thermionic-gun-based injector for a compact free-electron laser, Phys. Rev. Accel. Beams 20, 080702 (2017).

[24] M. Sands, The physics of electron storage rings: An introduction, SLAC Report No. 121, 1970.

[25] H. Wiedemann, Particle Accelerator Physics, 4th ed. (Springer International publishing, New York, 2015).

[26] M. Borland, Simple method for particle tracking with coherent synchrotron radiation, Phys. Rev. ST Accel. Beams 4, 070701 (2001). 